\title{
A Specification Language for the WIDE Workflow Model
}

\author{
Daniel K.C. Chan ${ }^{\dagger} \quad$ Jochem Vonk $^{\dagger} \quad$ Gabriel Sánchez $^{\dagger} \quad$ Paul W.P.J. Grefen ${ }^{\dagger} \quad$ Peter M.G. Apers ${ }^{\dagger}$ \\ ${ }^{\dagger}$ Computer Science Department, University of Twente, 7500 AE Enschede, The Netherlands \\ $\ddagger$ Sema Group sae, c/ Albaracin 25, 28037 Madrid, Spain \\ \{chan, vonk, grefen, apers\}@cs.utwente.nl, gsg@sema.es
}

\begin{abstract}
This paper presents a workflow specification language developed in the WIDE project. The language provides a rich organisation model, an information model including presentation details, and a sophisticated process model. Workflow application developers should find the language a useful and compact means to capture and investigate design details. Workflow system developers would discover the language a good vehicle to study the interaction between different features as well as facilitate the development of more advanced features. Others would attain a better understanding of the workflow paradigm and could use the language as a basis of evaluation for the functionality of workflow systems.
\end{abstract}

\section{Introduction}

There is a consensus that fundamental workflow management systems [7] need to be extended to meet the requirements of production workflow systems [5] and novel application domains [3]. Studies and discussions of such extensions have been hampered by the lack of a concrete framework. The aim of this paper is to introduce a concrete framework through the presentation of a workflow specification language. The language differs from other workflow languages and proposals in that it captures all the fundamental elements of the workflow paradigm (namely organisation model, information model, process model. and their relationships) at an abstraction level that is suitable for user development rather than machine manipulation. In other words, it provides a higher level of abstraction than many existing workflow languages.

The provision of a textual description for workflow applications is important for a number of reasons. If a workflow system is to be around for a considerable length of time. it may well require upgrading every now and then. Giren the rapid development in workflow products. upgrading maty be realised by switching to a different implementation plat form or worklow engine. A specification language can pase the transition in both circumstances by providing a bridge between different systems. Besides being part of a workflow

Permission to make digital/hard copy of all or part of this work for personal or classroom use is granted without fee provided that copies are not made or distributed for protit or commercial advantage. the copyright notice. the title of the publication and its date appear. and notice is given that copying is by permission of ACM. Inc. To copy otherwise, to republish. to post on servers or to redistribute to lists. requires prior specific permission and/or a fee C 1998 ACM 0.89791-969-6/98/0002 3.50 system, a specification language can also be used for process documentation.

The specification language presented in this paper has served as a vehicle for the development and synthesis of advanced workflow concepts within the ESPRIT WIDE project (see http://www.sema.es/projects/WDE/ for further information) [2]. The aim of the project is to deliver a commercial strength advanced workflow system to replace the FORO workflow system currently marketed by the Sema Group.

\section{An Overview of the WIDE Model}

The WIDE workflow model, which is inspired by the workflow model used in FORO, is an extension of the reference workflow model proposed by the Workflow Management Coalition [7]. Unlike the reference workflow modei, it supports a rich organisation model, sophisticated activi: assignment constraints, dynamic control flows including the use of active rules, complex process structures, and workflow transactions. A summary of the three "sub-models" of the WIDE workflow model is given next. A complete description of the WIDE model can be found in [1].

Organisation model. It registers the organisation structure and resources of an enterprise. It records information about individual employees (staff), functional positions held by staff (position), groups of staff (team) that are put together to serve some business transaction, as well as nonhuman resources (tool) such as machines and software. A staff member can hold several positions as well as participate in a number of teams possibly in different capacities according to the positions held. Staff, positions, teams, and tools are collectively referred to as agents. All agents are associated with a domain which is usually used to model geographical sites or functional units of an organisation. The deputy relationship allows one staff member to deputise for another that is not available to carry out an activity. The position hierarchy captures the accountability relationship between positions. When a staff member is not available, a task can be performed instead by his or her senior to whom the staff member is accountable for. The team hierarchy recording the inclusion relationship serves a similar purpose. When a team inember is not available, the team leader can take over. However, if no one in the team is available, the leader of the affliated team which includes the original team can be brought in.

Information Model. It defines the data used in a workHow process. governs the operations that can be performed on the data. as well as controls the scope and presentation of thr data. Data can have one of two possible scopes: 
global and local. Global data are shared by all workflow processes. They are persistent and are stored usually in either databases or files. Local data are only accessible from within one workflow process and are shared between activities within the same process. The entity data type is introduced to capture external data and their associated operations. Every entity may have a set of associated operations that are often completely different from any other entities. The form data type is probably the most distinguishing type in the information model. It resembles the record type but with presentation information which is important due to the interactive nature of workflow systems. Each field in a form is typed and can be used to show or update data variables. Default field values can be defined. A form type can be derived from another form type possibly by removing fields. Moreover a derived form can relax or restrict operations that can be performed on a field. This mechanism encourages and supports re-usability which reduces the maintenance effort.

Process Model. The use of activity abstraction in the process model allows an application to be defined using topdown refinement which makes the ordeal more manageable and the resultant design more comprehensible. In other words, an activity representing a unit of work at one level can be refined into a network of activities in the next level. Activities at the bottom level, so to speak, are the building blocks of the process where actions are actually carried out. The bottom-level activities are referred to as base tasks while other activities are called tasks. This notion of activity abstraction is exploited in the transactional support within the WIDE model [6]. Every activity definition can contain the following components: (1) a pre-condition that has to be satisfied for the activity to start, (2) actions performed by the activity, (3) a post-condition that has to hold for the activity to terminate, (4) a role constraint about assigning the activity to an agent and making it observable to an agent, (5) a schedule for the execution of the activity and its priority, and (6) handlers for system-defined as well as user-defined exceptions. The action part of an activity varies depending on whether the activity is a task or a base task. For a task it captures the control flows between activities in the next level. For a base task it captures some computation which may be performed over the information model or some external operation performed by the user. Consequently, role constraints may differ between tasks and base tasks. For a task a role constraint may specify dependency between activity assignments of lower-level activities. For a base task a role constraint is always about the base task alone. Base tasks have the option of specifying the forms that can be used for interaction with the user

\section{Example Specifications}

The example used in this section is about an hypothetical application about publishing technical papers produced within the WIDE project. Before a paper is submitted for publication, an internal review is carried out. If the result of the review is favourable, the paper will be submitted. Otherwise, the paper will be rewritten and reviewed again. The rewrite and review cycle can iterate for a number of times before the paper is submitted. Specification of forms: and prucesses is presented here. Specification of data and organisation is not presented here but can be foumd in $\mid 4$.

Form Specification. A form specification ematrins [re]resentation details concerning the display of a form and the trpes of the data that can appear in a form. A ropieal example is the "Review" form defined in line 2-14 in Figne 1.

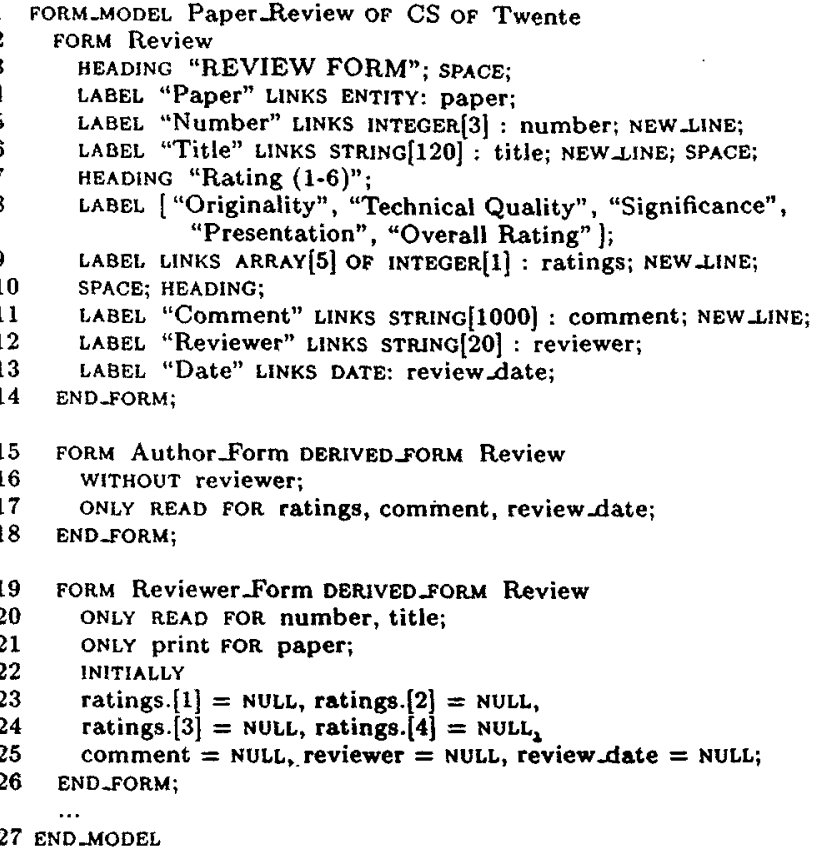

Figure 1: Form Model - Publication Process

The heading statement; (line 3,7,10) mark a new formatting section and option? ly print a string on the screen (line 3,7 ). While NEW_LINE and SPACE correspond to starting a new line and producing some vertical spacing. A LABEL statement usually generates a number of labelled boxes (or menus) though either the boxes (line 8) or the labels (line 9) can be omitted. It should be noted that fields in a form are references to local data and they are not value holders themselves.

The "Author Form" is derived from the "Review" form and differs from the latter in two ways. First, the field "re viewer" is removed (line 16). Second, the fields "ratings" "comment", and "review date" cannot be changed (line 17) Similarly, "Reviewer Form" is derived from the "Review" form. It restricts access to field "paper" to only the user defined operation "print" (line 21). Initial values are alse given for other fields (line 23-25) where NULL is a commor value for all types. Derived forms are therefore used to con trol the scope and access of local data by means of structura changes, access constraints, and initial values for fields be fore the form is displayed.

Process Specification. The publication process be gins with importing an organisation model (line 2), a forn model (line 3), and a data model (line 4) relevant to the ap plication. The control part, line 5-13, specifies the top leve activity abstraction. The workflow starts with the "Sub nit Paper" task (line 6) and finishes with either the "Re reive.Review" task (line 12) or the two tasks "Polish_Paper and "Solicit_Funding" (line 13). The transition from on task to another is captured using the ENABLE statemer. (line 7 shows its simplest form). Conditional transitions ca be sperified as in line 8 and 10. The task "Polish.Paper is refined in lin" 26-30. The total join from "Write_Paper 


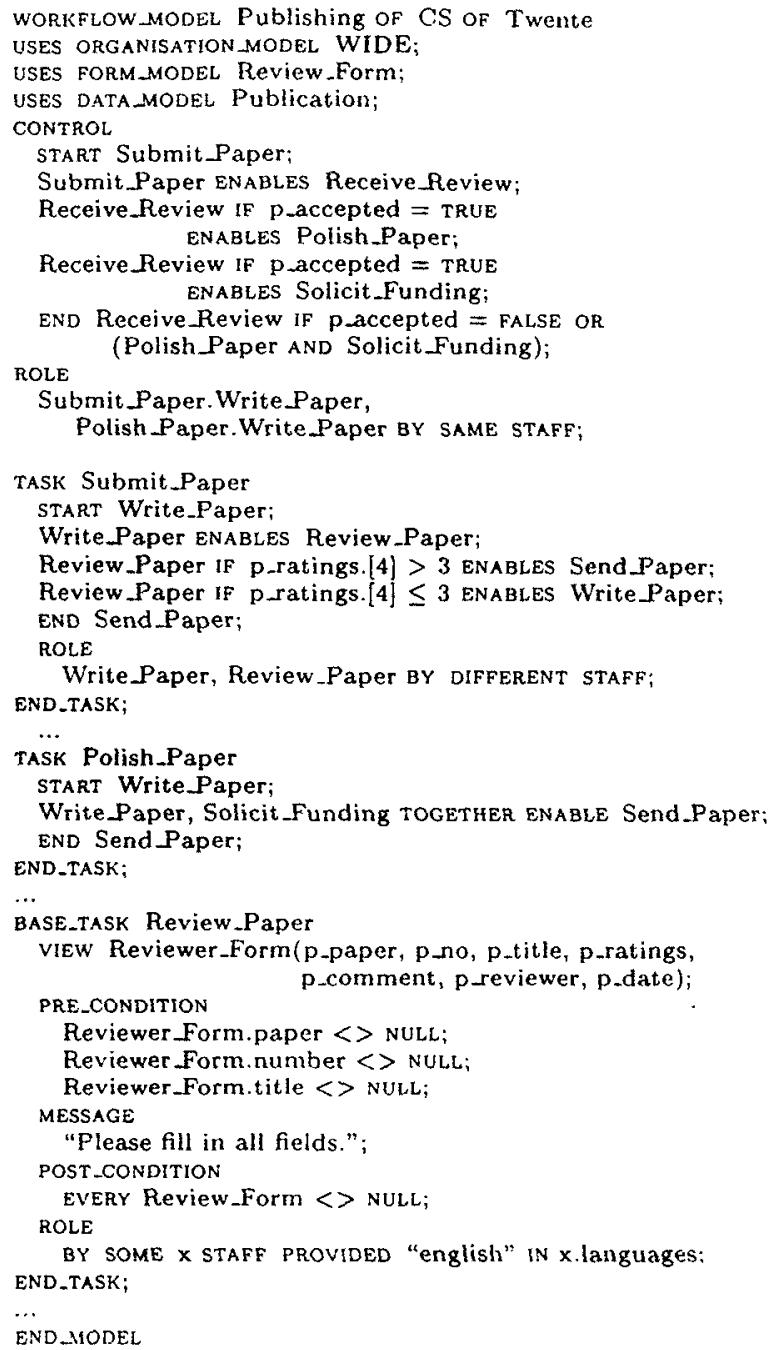

Figure 2: Process Model - Publication Process

and "Solicit_Funding" to "Send_Paper" is captured using the keyword TOGETHER ENABLE.

In the case of a base task, instead of control statement. computation and message display are specified (line $37-38$ ) The message may be displayed together with other form. Forms are displayed only by base tasks which have the dutv of performing computation that may require inputs from the user. The forms to be displayed is specified in the YIEW clause and their contents are filled using local variables (line $32)$. Every activity can be given a pre-condition and a posicondition that have to be satisfied for it to begin and end successfully. The pre-condition of "Review_Paper" given in line 33-36 specifies that some fields in the form must be fillext The post-condition given in line $39-40$ sperifies that all the. fields must be filled. The kerword Evtry is a shorthand fo: all the fiolds in a form

Activity Assignment. Rule ronstraints are used guvern the assignment of activities to agents. Com-tame -

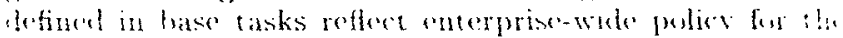

activities, The one given in line $41-42$ in Figure 2 specifies that base task "Review Paper" is to be performed by someone who knows "english". When activities are put together to form an application, additional constraints are usually required to dictate relationships between activities. The constraint specified in line 23-24 insists that the author cannot review his or her own paper while the constraint in line 14-16 requires that the person who writes the paper to be responsible for its polishing.

\section{Conclusions}

This paper presented the WIDE workflow model and a useroriented specification language for the model. The specification language differs from existing workflow specification languages and proposals in that all fundamental elements of the workflow paradigm are captured and elegantly integrated. Distinctive features include use of possibly partially parameterised domains, combination of specifications, incorporation of presentation details in form specifications, reusability over form specifications, declarative activity assignment, declarative exception handling, and workflow transactions. The specification language forms the basis of the current WIDE specification language whose implementation has just been completed.

\section{References}

11) F. Casati, P. Grefen, B. Pernici, G. Pozzi, and G. Sánchez. WIDE Workflow Model and Architecture. Technical Report 96-19, Centre for Telematics and Information Technology (CTIT), University of Twente, Netherlands, 1996.

(2) S. Ceri, P. Grefen, and G. Sánchez. WIDE - A Distributed Architecture for Workflow Management. In Proceedings of the International Workshop on Research Issues in Data Engineering, pages 76-79. IEEE Computer Society Press, 1997.

[3] D.K.C. Chan and K.R.P.H. Leung. A Workflow Vista of the Soft ware Process. In Proceedings of the International Workshop on Database and Expert Systems Applications, pages 62-67. IEEE Computer Society Press, 1997.

[4] D.K.C. Chan, J. Vonk, G. Sánchez, P.W.P.J. Grefen, and P.M.G. Apers. A Conceptual Workflow Specification Language. Technical Report 96-48, Centre for Telematics and Information Technology (CTIT), University of Twente. Cetherlands, 1996.

[5] D. Georgakopoulos and M. Hornick. An Overview of Workflow Management: from Process Modeling to Norkflow Automation Infrastructure. Distributed and Parnllel Databases, 3(2):119-153, 1995.

[6] P. Grefen, J. Vonk, E. Boertjes, and P. Apers. TwoLaver Transaction Management for Workflow Managemont Applications. In Proceedings of the International Confrtrtut on Database and Expert Systems Applica. homs. volume 1308 of Lecture Notes in Computer Sci. th.. pages 430 439. Springer-Verlag, 1997.

$\because$ Trmminolugy \& Glossary. Technical Report TC-1011, Winkflow Mathigrment Coalition, June 1996. 